\title{
Association of Nondiabetic Glucometabolic Status and Aortic Stiffness in Community Hypertension Patients
}

\author{
Dan Zhou (D)', Mengqi Yan', Songtao Tang², Yingqing Feng' \\ 'Department of Cardiology, Guangdong Cardiovascular Institute, Guangdong Provincial People's Hospital, Guangdong Academy of Medical Sciences, \\ Guangzhou, 510080, People's Republic of China; ${ }^{2}$ Department of Internal Medicine, Community Health Center of Liaobu Community, Dongguan, \\ People's Republic of China
}

Correspondence: Yingqing Feng, Department of Cardiology, Guangdong Cardiovascular Institute, Guangdong Provincial People’s Hospital, Guangdong Academy of Medical Sciences, No. 106 Zhongshan Two Road, Yuexiu District, Guangzhou, 510080, People’s Republic of China, Email I250780250@qq.com

Background: Diabetes is most commonly associated with aortic stiffness, but the importance of nondiabetic glucometabolic status for aortic stiffness (AS) in hypertension patients is unclear.

Methods: We included 1065 hypertension patients without diabetes in a cohort study. Carotid-femoral pulse wave velocity (cfPWV) $>10 \mathrm{~m} / \mathrm{s}$ can broadly be defined as AS. Pearson correlation analysis and multiple regression analysis are used to reveal the relationship between elevated fasting blood glucose (FBG) and AS.

Results: The 1065 hypertension patients (mean age 60 years) included 48\% male, 22\% smokers, 94.3\% with anti-hypertensive drugs, $17.9 \%$ with AS, $80 \%$ with abdominal obesity, $42 \%$ with elevated triglycerides (TG), and $27 \%$ with elevated FBG. The mean values for office systolic blood pressure (SBP)/diastolic blood pressure (DBP) and central SBP/DBP were $130 / 85 \mathrm{mmHg}$ and $132 / 86 \mathrm{mmHg}$. Mean cfPWV was $8.7 \mathrm{~m} / \mathrm{s}$. Multiple regression analysis revealed that age, office SBP, and elevated FBG were independently related to AS in the whole hypertension. Elevated FBG had 1.6-fold risk of AS in hypertension patients compared with below the cutoff. In subgroup analysis, elevated FBG increased 2.68-fold risk for AS in those without metabolic syndrome (MS), not in MS. The area under curve (AUC) of office SBP was higher than central SBP for AS in receiver operating characteristic (ROC) analysis.

Conclusion: We found that elevated FBG was an independent risk factor for AS in hypertension patients without MS, although there was a high proportion of abdominal obesity. Office SBP was better than central SBP to assess AS in community hypertension.

Keywords: aortic stiffness, hypertension, elevated plasma blood glucose, carotid-femoral pulse wave velocity

\section{Introduction}

Hypertension is a contributing factor for cardiovascular complications such as stroke, myocardial infarction, renal dysfunction and heart failure. ${ }^{1}$ Previous studies have confirmed aortic stiffness (AS) was related to adverse cardiovascular events and target organ damage in hypertension. In resistant hypertension, patients with increased AS had a significantly more than 2-fold increased risk of cardiovascular events and mortality. ${ }^{2}$ Regional AS as assessed by carotid-femoral pulse wave velocity (cfPWV) is associated with renal organ damage, ${ }^{3}$ greater basal left ventricular torsion and improved diastolic function ${ }^{4}$ in hypertensive patients.

Type 2 diabetes is most commonly associated with $\mathrm{AS},{ }^{5}$ but not impaired fasting glucose,${ }^{6}$ in apparently healthy populations. There is controversy over the association of $\mathrm{HbAlc}$ and AS in individuals without diabetes. ${ }^{7,8}$ Advanced glycation end products and increased fasting blood glucose (FBG) levels are associated with AS in individuals without diabetes. ${ }^{7}$ Hence, the conclusion between nondiabetic glucometabolic status and AS is inconsistent in non-diabetes. Hypertension patients have high prevalence of metabolic syndrome (MS) risk factors, ranging from 40 to $58 \%$ in 15 cities in China. ${ }^{9}$ The risk factors of MS were associated with brachial-ankle PWV (baPWV) in some general 
populations in China and Japan. ${ }^{10,11}$ The study aims to explore the association of nondiabetic glucometabolic status, elevated FBG, and AS in community hypertension patients with MS or not.

\section{Methods}

\section{Study Population}

This is a cross-sectional study that enrolled 1551 community hypertensive patients, who attended detailed annual examination in one community healthcare center in Guangdong province, China. Patients were recruited continuously from 1 May 2015 to 31 December 2016. Participants who had missing data on lipid results ( $n=147)$ or diagnosed with type 2 diabetes $(n=339)$, coronary heart disease $(n=32)$, or stroke $(n=30)$ were excluded from this study, leaving 586 patients with MS and 479 patients without MS for data analysis (Figure 1). The study protocol was approved by Ethics Committee of Guangdong Provincial People's Hospital. All procedures carried out were in line with the ethical standards of the responsible committees on human experimentation and with the Declaration of Helsinki. All participants have signed informed consent.

\section{Blood Pressure Measurement}

For included participants, seated office blood pressure (BP) and heart rate were obtained after 5 minutes of rest in the right arm of the participants with blood pressure monitor. Baseline BP values were obtained at three minute intervals. The average of last two consecutive recordings was recorded. Systolic BP (SBP) $\geq 140 \mathrm{~mm} \mathrm{Hg}$ and/or diastolic BP (DBP) $\geq 90 \mathrm{mmHg}$ and/or the use of antihypertensive medicine within 2 weeks are the definition of hypertension. ${ }^{12}$

\section{Data Collection}

Enrolled participants were administered a baseline questionnaire by trained research staff to collect demographic data, medication history, smoking status, drinking status and medical history. Body weight, height and waist circumference (WC) were measured in physical examination. Body mass index (BMI) was calculated using weight (kg) divided by the square of height $(\mathrm{m} 2)$.

\section{Laboratory Examinations}

Laboratory examinations were collected after at least 12 hours of fasting. FBG concentrations were measured by the glucose oxidation method. Triglycerides (TG), total cholesterol (TC), high-density lipoprotein (HDL) and low-density

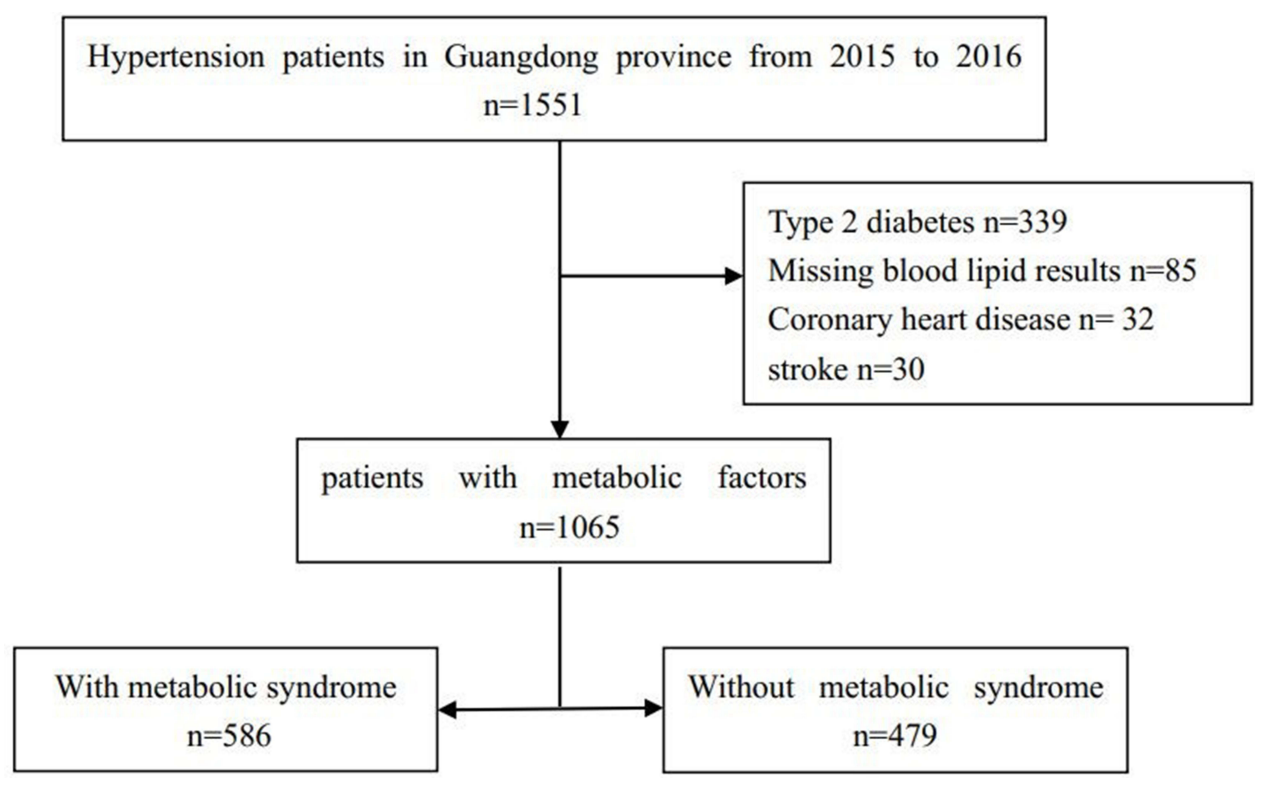

Figure I Study flow chart. 
lipoprotein (LDL) cholesterol concentrations were measured by enzymatic methods. Renal function was evaluated by e-GFR ${ }^{13}$ using serum creatinine. Serum uric acid (UA) was measured by the Uricase-POD method on an automated analyzer.

\section{Metabolic Syndrome}

Metabolic syndrome is defined as presence of any 3 of the following 5 elements. ${ }^{14}$ Abdominal obesity was defined as $\mathrm{WC} \geq 85 \mathrm{~cm}$ (men) or $\geq 80$ (women). Elevated TG was defined as TG $\geq 150 \mathrm{mg} / \mathrm{dL}$. Reduced HDL-C was defined as HDL$\mathrm{C}<40 \mathrm{mg} / \mathrm{dL}$ (men) or HDL-C $<50 \mathrm{mg} / \mathrm{dL}$ (women). Elevated BP (antihypertensive drug treatment in a patient with a history of hypertension is an alternative indicator) was defined as SBP $\geq 130$ and/or DBP $\geq 85 \mathrm{~mm} \mathrm{Hg}$. Elevated FBG was defined as FBG $\geq 100 \mathrm{mg} / \mathrm{dL}$.

\section{Central BP Measurements and Pulse Wave Velocity (PWV)}

We use a widely validated system (ABI-system 100; Bosch +Sohn GmbH U. Co. KG) and analysis software to calculate pressure waves. After a 30-minute rest in a supine position, pulse wave recordings were performed consecutively at the right carotid artery; previous study indicated central augmentation index (AI) may be more precise in this vascular area. ${ }^{15}$ We confirmed pulse wave recordings if the variation of the peak and bottom values of single waves was $<5 \%$ or took the measure again. Central pulse waves were also analyzed to obtain augmentation pressure (AP) and AI as (AP/pulse pressure (PP)) x 100. Aortic PWV was determined by carotid and femoral artery pressure waveforms. From carotid and femoral artery pressure waveforms, carotid to femoral transit time (DT) was calculated. The path length (L) between the carotid and femoral arteries was determined by the landmark of the sternal notch and femoral artery, and PWV calculated as L/DT. ${ }^{16}$ Carotid-femoral PWV (cfPWV), a threshold of $>10 \mathrm{~m} / \mathrm{s}$, was the gold standard to assess AS. ${ }^{17,18}$

\section{Statistical Analysis}

Participants were categorized into 5 subgroups based on the risk factors of MS: group 1: 1 risk factor of MS; group 2: 2 risk factors of MS; group 3: 3 risk factors of MS; group 4: 4 risk factors of MS; group 5: 5 risk factors of MS. Conventional cardiovascular risk factors were compared across the 5 subgroups using analysis of one-way ANOVA for continuous variables and $\chi^{2}$ tests for categorical variables. Risk factors of MS, peripheral hemodynamic parameters and central hemodynamic parameters were compared across the 5 groups. Correlations between cfPWV and metabolic risk factors and BP parameters was conducted by Pearson correlation analysis. Univariate and multivariate logistic regression were used to probe the relationship between the risk factors of MS and AS in hypertension patients. The confounding factors included age, the risk factors of metabolic syndrome, sex, smoking, office SBP, office DBP, central SBP, central DBP, AI, AP, eGFR, UA, abdominal obesity, elevated TG, reduced HDL-C, and elevated FBG. Central hemodynamic parameters and cfPWV were also compared between metabolic syndrome or not. Among MS, multivariate logistic regression was performed to compute odds ratios (ORs) of the elevated FBG for AS. In hypertension patients without MS, multivariate logistic regression was also used to assess elevated FBG for AS. Receiver operating characteristic (ROC) curves were built and the area under curve (AUC) was calculated to establish the ability of the office SBP and central SBP to assess the AS. All P-values were 2-tailed, and a significance level of 0.05 was used. All statistical analyses were conducted using SPSS 25.0 (SPSS Inc, Chicago, Illinois, USA).

\section{Results}

Among 1065 hypertension patients enrolled, 586 participants were MS, 479 were not. Table 1 presents the baseline characteristics of the study population according to the number of risk factors in hypertension patients. The 1065 hypertension patients (mean age 60 years) included $48 \%$ male, $22 \%$ smokers, and $94.3 \%$ with anti-hypertensive drugs, $17.9 \%$ with AS, $80 \%$ with abdominal obesity, $42 \%$ with elevated TG, and $27 \%$ with elevated FBG. Mean office SBP/ DBP, central SBP/DBP were $130 / 85 \mathrm{mmHg}$ and $132 / 86 \mathrm{mmHg}$. Mean cfPWV was $8.7 \mathrm{~m} / \mathrm{s}$. There was a significant difference between age, sex, BMI, WC, FBG, TG, HDL-C, UA, HR, and central DBP, the risk factors of MS among the 5 subgroups. AI and cfPWV did not show a difference between the 5 groups. Pearson correlation analysis, shown in Table 2, age, office SBP, central SBP, and AP showed positively related to cfPWV in hypertension patients (all $\mathrm{p}<0.05$ ). 
Table I Baseline Characteristics of the Study Population According to the Increased Risk Factors of Metabolic Syndrome in Hypertension Patients

\begin{tabular}{|c|c|c|c|c|c|c|c|}
\hline & $\begin{array}{l}\text { All } \\
n=1065\end{array}$ & $\begin{array}{l}\text { Group I, } \\
\mathrm{n}=\mathbf{8 0}\end{array}$ & $\begin{array}{l}\text { Group 2, } \\
n=399\end{array}$ & $\begin{array}{l}\text { Group 3, } \\
n=387\end{array}$ & $\begin{array}{l}\text { Group 4, } \\
n=172\end{array}$ & $\begin{array}{l}\text { Group 5, } \\
n=27\end{array}$ & p-value \\
\hline Age (years) & $60 \pm 11$ & $63 \pm 12$ & $61 \pm 11$ & $59 \pm 11$ & $59 \pm 11$ & $57 \pm 11$ & 0.001 \\
\hline Male, n (\%) & $518(48)$ & $60(75)$ & $199(50)$ & $182(47)$ & $69(40)$ & $8(29)$ & $<0.001$ \\
\hline Current smoking, n (\%) & $237(22)$ & $16(20)$ & $90(22)$ & $90(23)$ & $33(19)$ & $8(29)$ & 0.556 \\
\hline Current drinking, n (\%) & $103(9)$ & $6(7)$ & $33(8)$ & $44(1 \mathrm{I})$ & $19(11)$ & I (3) & 0.409 \\
\hline BMI (kg/m2) & $25.8 \pm 3$ & $21.8 \pm 4$ & $25.9 \pm 3$ & $26.2 \pm 3$ & $26.4 \pm 3$ & $26.6 \pm 3$ & $<0.001$ \\
\hline$W C(\mathrm{~cm})$ & $90 \pm 9$ & $77 \pm 5$ & $90 \pm 2$ & $91 \pm 9$ & $92 \pm 7$ & $92 \pm 6$ & $<0.001$ \\
\hline FBG $(\mathrm{mg} / \mathrm{dL})$ & $94 \pm 16$ & $87 \pm 7$ & $89 \pm 9$ & $96 \pm 18$ & $103 \pm 18$ & $111 \pm 12$ & $<0.001$ \\
\hline $\mathrm{TC}(\mathrm{mg} / \mathrm{dL})$ & $206 \pm 43$ & $206 \pm 41$ & $206 \pm 41$ & $206 \pm 44$ & $206 \pm 45$ & $194 \pm 38$ & 0.698 \\
\hline TG (mg/dL) & $165 \pm 132$ & $97 \pm 25$ & $120 \pm 85$ & $186 \pm 157$ & $234 \pm 142$ & $237 \pm 79$ & $<0.001$ \\
\hline HDL-C (mg/dL) & $54 \pm 12$ & $56 \pm 9$ & $58 \pm 11$ & $53 \pm 13$ & $48 \pm 12$ & $43 \pm 5$ & $<0.001$ \\
\hline LDL-C (mg/dL) & $124 \pm 33$ & $125 \pm 32$ & $124 \pm 35$ & $124 \pm 32$ & $123 \pm 37$ & $115 \pm 26$ & 0.683 \\
\hline eGFR (mL/min per $1.73 \mathrm{~m} 2)$ & $87 \pm 21$ & $84 \pm 21$ & $86 \pm 20$ & $89 \pm 23$ & $89 \pm 21$ & $91 \pm 24$ & 0.145 \\
\hline $\mathrm{UA}(\mu \mathrm{mol} / \mathrm{L})$ & $435 \pm 116$ & $454 \pm 102$ & $420 \pm 113$ & $440 \pm 121$ & $450 \pm 116$ & $434 \pm 91$ & 0.011 \\
\hline Antihypertensive drugs, n (\%) & $1017(94.3)$ & $70(87.5)$ & $374(93.7)$ & $371(95.8)$ & $170(98.8)$ & $26(96.2)$ & 0.084 \\
\hline \multicolumn{8}{|l|}{ Metabolic disorders } \\
\hline Abdominal obesity (\%) & $86 I(80)$ & 0 & $318(79.6)$ & $342(88.3)$ & $170(98.8)$ & $27(100)$ & $<0.001$ \\
\hline Elevated TG (\%) & $449(42)$ & 0 & $43(10.7)$ & $216(55.8)$ & $163(94.7)$ & $27(100)$ & $<0.001$ \\
\hline Reduced HDL-C (\%) & $228(21)$ & 0 & $10(2.5)$ & $93(24)$ & $98(56.9)$ & $27(100)$ & $<0.001$ \\
\hline Elevated FBG (\%) & $297(27)$ & 0 & $28(7)$ & $|3|(33.8)$ & III (64.5) & $27(100)$ & $<0.001$ \\
\hline \multicolumn{8}{|c|}{ Peripheral hemodynamic parameters } \\
\hline Heart rate, bpm & $70 \pm 10$ & $66 \pm 10$ & $69 \pm 10$ & $71 \pm 11$ & $71 \pm 11$ & $73 \pm 11$ & $<0.001$ \\
\hline SBP, mmHg & $140 \pm 15$ & $137 \pm 18$ & $141 \pm 15$ & $140 \pm 15$ & $139 \pm 16$ & $142 \pm 17$ & 0.352 \\
\hline $\mathrm{DBP}, \mathrm{mmHg}$ & $85 \pm 10$ & $82 \pm 9$ & $84 \pm 10$ & $86 \pm 10$ & $84 \pm 10$ & $85 \pm 10$ & 0.040 \\
\hline \multicolumn{8}{|c|}{ Central hemodynamic parameters } \\
\hline Central SBP, mmHg & $132 \pm 35$ & $128 \pm 15$ & $132 \pm 19$ & $131 \pm 18$ & $135 \pm 75$ & $132 \pm 17$ & 0.576 \\
\hline Central DBP, $\mathrm{mmHg}$ & $86 \pm 10$ & $83 \pm 10$ & $85 \pm 10$ & $87 \pm 10$ & $86 \pm 11$ & $86 \pm 10$ & 0.040 \\
\hline Central PP, $\mathrm{mmHg}$ & $45 \pm 14$ & $44 \pm 14$ & $46 \pm 15$ & $44 \pm 14$ & $44 \pm 14$ & $45 \pm 10$ & 0.158 \\
\hline $\mathrm{AP}, \mathrm{mmHg}$ & $14 \pm 8$ & $14 \pm 7$ & $15 \pm 8$ & $14 \pm 8$ & $14 \pm 8$ & $19 \pm 9$ & 0.258 \\
\hline $\mathrm{Al}, \%$ & $0.3 I \pm 0.1 I$ & $0.30 \pm 0.11$ & $0.31 \pm 0.11$ & $0.30 \pm 0.10$ & $0.31 \pm 0.1 I$ & $0.3 I \pm 0.14$ & 0.691 \\
\hline $\mathrm{cfPWV}, \mathrm{m} / \mathrm{s}$ & $8.7 \pm 3.6$ & $8.5 \pm 1.5$ & $8.6 \pm 1.7$ & $8.8 \pm 5.6$ & $8.6 \pm 1.7$ & $8.8 \pm 1.8$ & 0.899 \\
\hline cfPWV > $10 \mathrm{~m} / \mathrm{s}(\%)$ & $191(17.9)$ & $9(11)$ & $80(20)$ & $66(17)$ & $28(16)$ & $8(29)$ & 0.135 \\
\hline
\end{tabular}

Notes: Data was showed by mean \pm SD or $\mathrm{n}(\%)$. No. of risk factors, Group I: I risk factor of metabolic syndrome; Group 2: 2 risk factors of metabolic syndrome; Group 3: 3 risk factors of metabolic syndrome; Group 4: 4 risk factors of metabolic syndrome; Group 5: 5 risk factors of metabolic syndrome.

Abbreviations: BMI, body mass index; WC, waist circumference; FBG, plasma blood glucose; TC, total cholesterol; TG, triglycerides; HDL-C, high-density lipoprotein cholesterol; LDL-C, low-density lipoprotein cholesterol; UA, uric acid; SBP, systolic blood pressure; DBP, diastolic blood pressure; AP, augmentation pressure; Al, augmentation index; cfPWV, carotid-femoral pulse wave velocity.

Age and office SBP had a strong correlation with cfPWV ( $\mathrm{r}=0.219, \mathrm{r}=0.148$, respectively). In Supplement Figure $1 \mathrm{~A}-\mathrm{C}$, the results show older subjects had higher cfPWV and higher proportion of AS. Central SBP had a weak correlation with cfPWV (r=0.072).

In univariate logistic regression, age, office SBP, and elevated FBG were risk factors for AS in hypertension patients in Model 1 (Table 3) (all p <0.05). Central SBP and central DBP were not risk factors for cfPWV. In Model 2, age, office SBP and risk factors of MS still showed independently associated with AS after adjusted for age, risk factors of MS, sex, smoking, office SBP, office DBP, central SBP, central DBP, AI, AP, eGFR, and UA (all $\mathrm{p}<0.05$ ). In Model 3, elevated FBG became an independent risk factor for aortic stiffness (OR (95\% CI), 1.623 (1.131-2.329)), after we added abdominal obesity, elevated TG, reduced HDL-C and elevated FBG.

In Table 4, the results show no difference between central BP, AP, AI, and cfPWV among MS or not (all $\mathrm{p}>0.05)$. The mean cfPWV in MS or without MS was $8.8 \mathrm{~m} / \mathrm{s}$ and $8.6 \mathrm{~m} / \mathrm{s}$, respectively. The presence of AS was $17.4 \%$ and $18.5 \%$, respectively. We explored the relationship between the elevated FBG and aortic stiffness in MS in Table 5. In a univariate 
Table 2 Correlations Between cfPWV and Metabolic Risk Factors and Blood Pressure Parameters

\begin{tabular}{|l|l|l|}
\hline & $\boldsymbol{r}$ & p-value \\
\hline Age (years) & 0.219 & 0.000 \\
BMI $(\mathrm{kg} / \mathrm{m} 2)$ & 0.001 & 0.976 \\
WC $(\mathrm{cm})$ & 0.000 & 0.998 \\
FBG $(\mathrm{mg} / \mathrm{dL})$ & 0.02 & 0.515 \\
TC $(\mathrm{mg} / \mathrm{dL})$ & -0.003 & 0.929 \\
TG $(\mathrm{mg} / \mathrm{dL})$ & -0.002 & 0.955 \\
HDL-C $(\mathrm{mg} / \mathrm{dL})$ & -0.001 & 0.977 \\
LDL-C $(\mathrm{mg} / \mathrm{dL})$ & $-0.01 \mathrm{1}$ & 0.726 \\
eGFR $(\mathrm{mL} / \mathrm{min}$ per I.73 m2) & -0.102 & 0.001 \\
UA ( $\mu \mathrm{mol} / \mathrm{L})$ & -0.006 & 0.846 \\
Peripheral hemodynamic parameters & & \\
SBP, mmHg & 0.148 & 0.000 \\
DBP, mmHg & 0.036 & 0.247 \\
Central hemodynamic parameters & & \\
Central SBP, mmHg & 0.072 & 0.019 \\
Central DBP, mmHg & 0.038 & 0.215 \\
AP, mmHg & 0.103 & 0.001 \\
Al, \% & 0.038 & 0.219 \\
\hline
\end{tabular}

Abbreviations: BMI, body mass index; WC, waist circumference; FBG, plasma blood glucose; TC, total cholesterol; TG, triglycerides; HDL-C, high-density lipoprotein cholesterol; LDL-C, low-density lipoprotein cholesterol; UA, uric acid; SBP, systolic blood pressure; DBP, diastolic blood pressure; AP, augmentation pressure; Al, augmentation index; cfPWV, carotid-femoral pulse wave velocity.

Table 3 Multivariate Logistic Regression Analysis of Aortic Stiffness in Hypertension Patients

\begin{tabular}{|c|c|c|c|}
\hline & $\begin{array}{l}\text { Aortic Stiffness } \\
\text { OR }(95 \% \mathrm{CI}) \text { Model I }\end{array}$ & Model 2 & Model 3 \\
\hline Age & I.08I (I.063-I.098) & I.09I (I.07I-I.III) & $1.089(1.069-1.109)$ \\
\hline SBP & $1.026(1.017-1.034)$ & $1.033(1.02 I-1.045)$ & $1.033(1.021-1.045)$ \\
\hline Elevated FBG & 1.575 (1.128-2.199) & NS & $1.623(1.131-2.329)$ \\
\hline Group 5 & Reference & Reference & NS \\
\hline Group 4 & $0.298(0.10 \mathrm{I}-0.88 \mathrm{I})$ & $0.153(0.047-0.497)$ & NS \\
\hline Group 3 & $0.573(0.24 I-1.366)$ & $0.336(0.131-0.864)$ & NS \\
\hline Group 2 & $0.466(0.194-1.116)$ & $0.316(0.123-0.816)$ & NS \\
\hline Group I & $0.420(0.166-1.060)$ & $0.297(0.109-0.806)$ & NS \\
\hline
\end{tabular}

Notes: No. of risk factors, Group I: I risk factor of metabolic syndrome; Group 2: 2 risk factors of metabolic syndrome; Group 3: 3 risk factors of metabolic syndrome; Group 4: 4 risk factors of metabolic syndrome; Group 5: 5 risk factors of metabolic syndrome; Model I: not adjusted. Model 2: adjusted for age, risk factor of metabolic syndrome, sex, smoking, SBP, DBP, central SBP, central DBP, Al, AP, eGFR, and UA.

logistic regression, age, office SBP, elevated FBG and increased risk factors of MS were risk factors for aortic stiffness in Model 1 (all p <0.05). In Model 3, compared with group 5, the ORs (95\% CI) were $0.233(0.087-0.626)$ and 0.237 (0.085-0.661) for group 4 and group 3 after adjusted for age, increased risk factors of MS, sex, smoking, office SBP, office DBP, central SBP, central DBP, AI, AP, eGFR, UA, abdominal obesity, elevated TG, reduced HDL-C, and elevated FBG, and then elevated FBG was not the independent risk factor. In Table 6, relationship between elevated FBG and AS in hypertension without MS is presented. In a univariate logistic regression, age, office SBP and elevated FBG were risk factors for AS in Model 1 (all $\mathrm{p}<0.05$ ). In Model 3, among the metabolic risk factors, elevated FBG was still the independent risk factor for AS. Elevated FBG showed 2.68-fold risk for AS. 
Table 4 Relationship Between Central Hemodynamic Parameters and Metabolic Syndrome

\begin{tabular}{|l|l|l|l|}
\hline & $\begin{array}{l}\text { Metabolic Syndrome, } \\
\mathbf{n = 5 8 6}\end{array}$ & $\begin{array}{l}\text { Without Metabolic } \\
\text { Syndrome, =479 }\end{array}$ & p-value \\
\hline Central SBP, mmHg & $132 \pm 44$ & $131 \pm 19$ & 0.429 \\
Central DBP, mmHg & $86 \pm 10$ & $85 \pm 10$ & 0.918 \\
Central PP, mmHg & $44 \pm 14$ & $46 \pm 15$ & $0.45 \mathrm{I}$ \\
AP, $\mathrm{mmHg}$ & $14 \pm 8$ & $15 \pm 8$ & 0.408 \\
$\mathrm{Al}, \%$ & $0.30 \pm 0.1 \mathrm{I}$ & $0.31 \pm 0.1 \mathrm{I}$ & 0.900 \\
$\mathrm{cfPWV}, \mathrm{m} / \mathrm{s}$ & $8.8 \pm 4.6$ & $8.6 \pm 1.6$ & 0.342 \\
$\mathrm{cfPWV}>\mathrm{IO} \mathrm{m} / \mathrm{s}(\%)$ & $102(17.4)$ & $89(18.5)$ & 0.507 \\
\hline
\end{tabular}

Note: Data was showed by mean \pm SD or $n(\%)$.

Abbreviations: SBP, systolic blood pressure; DBP, diastolic blood pressure; AP, augmentation pressure; Al, augmentation index; cfPWV, carotidfemoral pulse wave velocity.

Table 5 Multivariate Logistic Regression Analysis Between Metabolic Risk Factors and Aortic Stiffness in Hypertension Patients with Metabolic Syndrome

\begin{tabular}{|c|c|c|c|}
\hline & $\begin{array}{l}\text { Aortic Stiffness OR }(95 \% \mathrm{Cl}) \\
\text { Model I }\end{array}$ & Model 2 & Model 3 \\
\hline Age & I.092 (I.067-I.II7) & $1.090(1.064-1.117)$ & 1.090 \\
\hline SBP & $1.034(1.022-1.046)$ & $1.029(1.016-1.042)$ & $1.029(1.016-1.042)$ \\
\hline Elevated FBG & $\mathrm{I} .66 \mathrm{I}(\mathrm{I} .08 \mathrm{I}-2.55 \mathrm{I})$ & NS & NS \\
\hline Group 5 & Reference & Reference & Reference \\
\hline Group 4 & $0.298(0.10 \mathrm{I}-0.88 \mathrm{I})$ & $0.290(0.111-0.759)$ & $0.290(0.111-0.759)$ \\
\hline Group 3 & $0.573(0.24 \mathrm{I}-\mathrm{I} .366)$ & $0.273(0.099-0.75 \mathrm{I})$ & $0.273(0.099-0.751)$ \\
\hline
\end{tabular}

Notes: Group 3: 3 components of metabolic syndrome; Group 4: 4 components of metabolic syndrome; Group 5: 5 components of metabolic syndrome; Model I: not adjusted. Model 2: adjusted for age, risk factors of metabolic syndrome, sex, smoking, SBP, DBP, central SBP, central DBP, AI, AP, eGFR, and UA. Model 3: adjusted for age, risk factors of metabolic syndrome, sex, smoking, SBP, DBP, central SBP, central DBP, Al, AP, eGFR, UA, abdominal obesity, elevated TG, reduced HDL-C, and elevated FBG.

Table 6 Multivariate Logistic Regression Analysis Between Metabolic Risk Factors and Aortic Stiffness in Hypertension Patients without Metabolic Syndrome

\begin{tabular}{|l|l|l|l|}
\hline & $\begin{array}{l}\text { Aortic Stiffness OR (95\% CI) } \\
\text { Model I }\end{array}$ & Model 2 & Model 3 \\
\hline Age & $1.069(1.044-1.094)$ & $1.078(1.052-1.106)$ & $1.077(1.050-1.105)$ \\
SBP & $1.016(1.004-1.029)$ & $1.019(1.005-1.032)$ & $1.019(1.005-1.033)$ \\
Elevated FBG & $3.226(1.44 I-7.220)$ & NS & $2.681(1.115-6.445)$ \\
\hline
\end{tabular}

Notes: Model I: not adjusted. Model 2: adjusted for age, sex, smoking, SBP, DBP, central SBP, central DBP, AI, AP, eGFR, and UA. Model 3: adjusted for age, sex, smoking, SBP, DBP, central SBP, central DBP, Al, AP, eGFR, UA, abdominal obesity, elevated TG, reduced HDL-C, and elevated FBG.

Office SBP was an independent risk factor for AS in all models in hypertension patients; however, central SBP was not. In Figure 2, the ROC analysis shows office SBP and central SBP have the ability to assess the AS ( $p<0.001)$. Office SBP was better than central SBP to assess AS in community hypertension.

\section{Discussion}

We found elevated FBG was a significant risk factor for AS in hypertension patients without MS. The increased metabolic risk factors were an independent risk factor for AS in MS. Patients with 4 metabolic risk factors and 3 metabolic risk factors had only 0.2 -fold risk for aortic stiffness compared with those with 5 metabolic risk factors. 


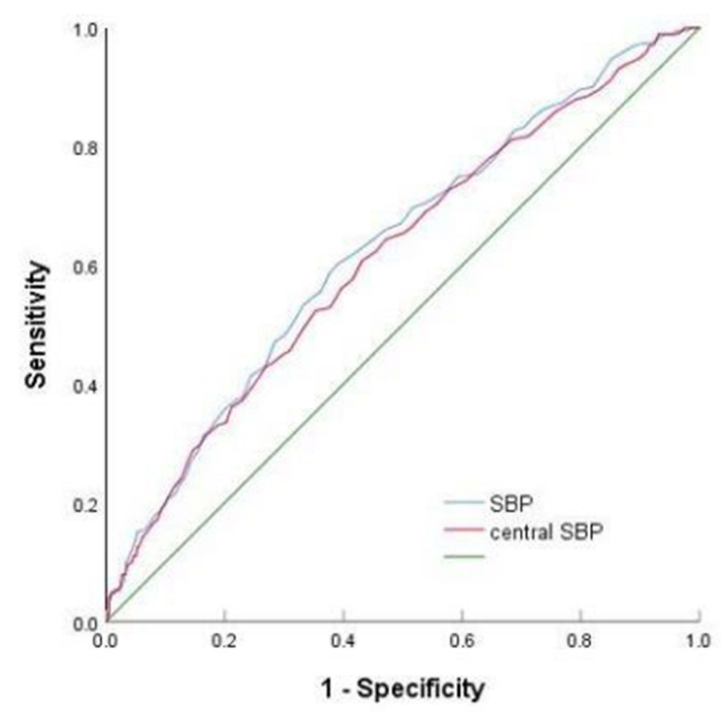

A

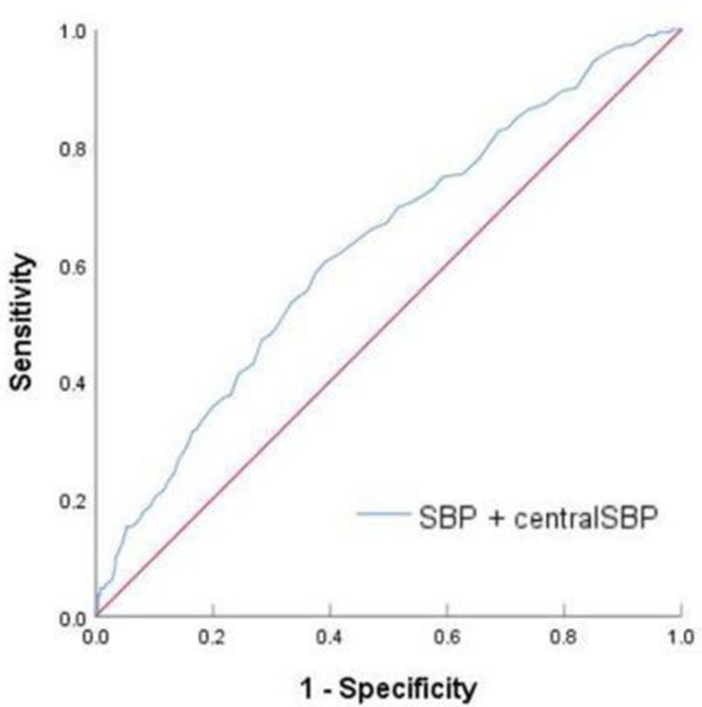

B

\begin{tabular}{lll}
\hline variate & AUC & p-value \\
\hline SBP & 0.629 & $<0.001$ \\
Central SBP & 0.615 & $<0.001$ \\
SBP + Central SBP & 0.629 & $<0.001$ \\
\hline
\end{tabular}

Figure 2 Receiver operating characteristic analysis for aortic stiffness by SBP and central SBP.

Elevated FBG presented a 2.68-fold risk for AS in non-MS participants, although a high proportion of these had abdominal obesity. Office SBP was better than central SBP to assess AS.

Elevated FBG had a 1.6-fold risk of AS in hypertension patients; when we adjusted other potential risk factors for stiffness, it only modestly changed the strength of association with elevated FBG. Previous studies showed similar results using various designs, indices of stiffness, and glucometabolic indices. Fasting glucose was associated with baseline cfPWV in 4386 participants without diabetes. ${ }^{7}$ One study, investigating two populations, ${ }^{19}$ showed fasting glucose was independently related to cfPWV in health volunteers and $\mathrm{HbAlc}$ was independently related to cfPWV in type 2 diabetes mellitus patients. Previous study revealed that FBG was associated with PWV in subjects with impaired fasting glucose ${ }^{20}$ In hypertension ${ }^{21,22}$ the SAGE score, based on the office SBP, age, FBG level, and eGFR, may be a striking marker for distinguishing subjects with elevated brachial-ankle PWV (baPWV) values. It was consistent with our results that age, office SBP and elevated FBG were the independent risk factors for AS in hypertension without diabetes.

In our subgroup analysis, we found elevated FBG remained an independent risk factor in non-MS patients. In the nonMS, although hypertension and abdominal obesity had high proportions, the results showed age, SBP and elevated-FBG were independent risk factors. Elevated-FBG presented a 2.68-fold risk for AS in non-MS. A $1 \mathrm{mmHg}$ higher office SBP was associated with a 1.019-fold risk increase for AS. Metabolic risk factors such as elevated-FBG had higher risk for AS than age or office SBP.

In MS patients, the combination of risk factors showed better correlation with AS. We found patients with 4 metabolic risk factors and 3 metabolic risk factors had a 0.2-fold risk for AS compared with those with 5 metabolic risk factors. Prior study indicated that clustered features of MS strikingly increased baPWV in Japanese men and 
women without coronary heart disease and stroke. ${ }^{11}$ The baPWV was associated with increment of metabolic risk factors in the community-based asymptomatic participants in China. ${ }^{10}$ Our report is consistent with a previous study indicating that a clustering of metabolic risk factors might accelerate the progression of AS in subjects with MS. During the follow-up, the increment in PWV was significantly higher in the group with three and more risk factors than in other groups. ${ }^{23}$

cfPWV and the proportion of AS showed no difference between MS and non-MS in our study. It was inconsistent with previous studies. ${ }^{10,11,24}$ Previous results showed baPWV was significantly higher in the subjects with MS in general population, in population with health examination in hospital or in community population without coronary heart disease and stroke. Presence of MS induces an increase of AS in untreated hypertensive patients independently from age and office SBP. ${ }^{25}$ This may partly explain the hypertension population in our study with a mean age of 60 years. Recent study from Alessandro Maloberti ${ }^{26}$ found the subjects with MS had higher cfPWV than non-MS in hypertension population; MS was not an independent determinant of PWVbut increased BP was. This was partly consistent with our results due to the subjects with MS were older, had higher SBP and higher proportion of type 2 diabetes. The discrepancy indicated that age and SBP are the most important risks for AS. MS was independently associated with AS in MS in hypertension without type 2 diabetes. In addition, participants with 1 or 2 metabolic risk factors were older than participants in other groups in our study. Office and central SBP showed no difference between the five groups. Previous study ${ }^{27}$ showed that subjects with MS present worse BP control in treated hypertension patients compared with those without MS and suggested that age and BP are the main determinants of PWV and its progression. The reason for the difference in BP control may be the discrepancy of medical conditions between hypertension unit in hospital of Italy and community center in one town of China.

Office SBP is better than central SBP to assess AS in multivariate logistic regression. Age and SBP have strong correlations with cfPWV and central SBP had a weak correlation with cfPWV. Previous study showed SBP and age were independent predictors of aortic PWV in the control subgroup, not central SBP in healthy controls. ${ }^{28}$ MS was associated with an increased AS in never-treated hypertension and main explanatory factors were age, SBP and albumin excretion rate. $^{29}$

We still have several limitations in our study. First, it is a cross-sectional study without data on the progression of cfPWV and did not allow cause-and-effect or mechanistic inferences. In addition, we did not collect HbAlc, which may possibly explain our observed associations with plasma glucose. Second, the sample is small in community and only enrolled hypertension patients in South China. Third, this study is performed in middle-aged to elderly patients with hypertension, so the results may not apply to other individuals. Age is closely related with AS, although we adjusted age in all models. Fourth, we did not collect the inflammation characteristics but these are also important to AS. Fifth, the proportions of ACEI, ARB, CCB, $\beta$-blockers and thiazide diuretics are 13.8, 56.7,50.9,12.8 and 11.9\%, respectively, in our study. Only $1.7 \%$ were prescribed single $\beta$-blockers; other subjects with $\beta$-blockers or thiazide diuretics were all prescribed second drugs such as angiotensin converting enzyme inhibitors, angiotensin receptor blocker or calcium channel blockers to control blood pressure. We cannot clarify the effect of $\beta$-blockers or thiazide diuretics on FBG for the interference from other drugs.

\section{Conclusions}

Elevated FBG is an independent risk factor for AS in hypertension patients with non-MS. The increasing MS risk factors are an independent risk factor for AS in MS. For hypertension patients in community, the elevated FBG is the first risk factor that we should address to decrease risk of aortic stiffness, except age and SBP. Central SBP is useless to assess AS in community hypertension. Compared with the complication of central SBP, office SBP is enough in community.

\section{Ethics Approval and Consent to Participate}

The experimental protocol was approved by Ethics Committee of Guangdong Provincial People's Hospital. Written informed consent was obtained from participants. 


\section{Funding}

This research was supported by Science and Technology Plan Program of Guangzhou (201803040012), the Key Area R\&D Program of Guangdong Province (No.2019B020227005), the Fundamental and Applied Basic Research Foundation Project of Guangdong Province (2020A1515010738), the Climbing plan of Guangdong Provincial People's Hospital (DFJH2020022), Guangdong Provincial Clinical Research Center for Cardiovascular Disease (2020B1111170011).

\section{Disclosure}

The authors declared that they have no competing interests.

\section{References}

1. Onuh JO, Qiu H. New progress on the study of aortic stiffness in age-related hypertension. J Hypertens. 2020;38(10):1871-1877. doi:10.1097/ HJH.0000000000002452

2. Cardoso CRL, Salles GC, Salles GF. Prognostic impact of aortic stiffness in patients with resistant hypertension. Hypertension. 2019;73 (3):728-735. doi:10.1161/HYPERTENSIONAHA.118.12367

3. Bruno RM, Cartoni G, Stea F, et al. Carotid and aortic stiffness in essential hypertension and their relation with target organ damage: the CATOD study. J Hypertens. 2017;35(2):310-318. doi:10.1097/HJH.0000000000001167

4. Gnakamene JB, Safar ME, Levy BI, Escoubet B. Left ventricular torsion associated with aortic stiffness in hypertension. J Am Heart Assoc. 2018;7 (5). doi:10.1161/JAHA.117.007427

5. Johansen NB, Charles M, Vistisen D, et al. Effect of intensive multifactorial treatment compared with routine care on aortic stiffness and central blood pressure among individuals with screen-detected type 2 diabetes: the ADDITION-Denmark study. Diabetes Care. 2012;35(11):2207-2214. doi: $10.2337 / \mathrm{dc} 12-0176$

6. Chirinos JA, Segers P, Gillebert TC, et al. Central pulse pressure and its hemodynamic determinants in middle-aged adults with impaired fasting glucose and diabetes: the Asklepios study. Diabetes Care. 2013;36(8):2359-2365. doi:10.2337/dc12-1463

7. McEniery CM, Wilkinson IB, Johansen NB, et al. Nondiabetic glucometabolic status and progression of aortic stiffness: the Whitehall II Study. Diabetes Care. 2017;40(4):599-606. doi:10.2337/dc16-1773

8. Schram MT, Schalkwijk CG, Bootsma AH, Fuller JH, Chaturvedi N, Stehouwer CD. Advanced glycation end products are associated with pulse pressure in type 1 diabetes: the EURODIAB Prospective Complications Study. Hypertension. 2005;46(1):232-237. doi:10.1161/01. HYP.0000164574.60279.ba

9. Xie K, Bao L, Jiang X, et al. The association of metabolic syndrome components and chronic kidney disease in patients with hypertension. Lipids Health Dis. 2019;18(1):229. doi:10.1186/s12944-019-1121-5

10. Wang A, Su Z, Liu X, et al. Brachial-ankle pulse wave velocity and metabolic syndrome in general population: the APAC study. BMC Cardiovasc Disord. 2016;16(1):228. doi:10.1186/s12872-016-0409-x

11. Nakanishi N, Shiraishi T, Wada M. Brachial-ankle pulse wave velocity and metabolic syndrome in a Japanese population: the Minoh study Hypertens Res. 2005;28(2):125-131. doi:10.1291/hypres.28.125

12. Liu LS. 2010 Chinese guidelines for the management of hypertension. Zhonghua Xin Xue Guan Bing Za Zhi. 2011;39(7):579-615.

13. Levey AS, Bosch JP, Lewis JB, Greene T, Rogers N, Roth D. A more accurate method to estimate glomerular filtration rate from serum creatinine: a new prediction equation. Modification of Diet in Renal Disease Study Group. Ann Intern Med. 1999;130(6):461-470. doi:10.7326/0003-4819130-6-199903160-00002

14. Alberti KG, Eckel RH, Grundy SM, et al. Harmonizing the metabolic syndrome: a joint interim statement of the International Diabetes Federation Task Force on Epidemiology and Prevention; National Heart, Lung, and Blood Institute; American Heart Association; World Heart Federation; International Atherosclerosis Society; and International Association for the Study of Obesity. Circulation. 2009;120(16):1640-1645. doi:10.1161/ CIRCULATIONAHA.109.192644

15. Perticone M, Maio R, Tassone EJ, et al. Insulin-resistance HCV infection-related affects vascular stiffness in normotensives. Atherosclerosis. 2015;238(1):108-112. doi:10.1016/j.atherosclerosis.2014.11.025

16. Chen $\mathrm{CH}$, Ting CT, Nussbacher A, et al. Validation of carotid artery tonometry as a means of estimating augmentation index of ascending aortic pressure. Hypertension. 1996;27(2):168-175. doi:10.1161/01.HYP.27.2.168

17. Covic A, Siriopol D. Pulse wave velocity ratio: the new "gold standard" for measuring arterial stiffness. Hypertension. 2015;65(2):289-290. doi:10.1161/HYPERTENSIONAHA.114.04678

18. Tanaka A, Tomiyama H, Maruhashi T, et al. Physiological diagnostic criteria for vascular failure. Hypertension. 2018;72(5):1060-1071. doi:10.1161/HYPERTENSIONAHA.118.11554

19. Kozakova M, Morizzo C, Fraser AG, Palombo C. Impact of glycemic control on aortic stiffness, left ventricular mass and diastolic longitudinal function in type 2 diabetes mellitus. Cardiovasc Diabetol. 2017;16(1):78. doi:10.1186/s12933-017-0557-z

20. Nam JS, Park JS, Cho MH, et al. The association between pulse wave velocity and metabolic syndrome and adiponectin in patients with impaired fasting glucose: cardiovascular risks and adiponectin in IFG. Diabetes Res Clin Pract. 2009;84(2):145-151. doi:10.1016/j.diabres.2009.02.002

21. Tomiyama H, Vlachopoulos C, Xaplanteris P, et al. Usefulness of the SAGE score to predict elevated values of brachial-ankle pulse wave velocity in Japanese subjects with hypertension. Hypertens Res. 2020;43(11):1284-1292. doi:10.1038/s41440-020-0472-7

22. Xaplanteris P, Vlachopoulos C, Protogerou AD, et al. A clinical score for prediction of elevated aortic stiffness: derivation and validation in 3943 hypertensive patients. J Hypertens. 2019;37(2):339-346. doi:10.1097/HJH.0000000000001904

23. Safar ME, Thomas F, Blacher J, et al. Metabolic syndrome and age-related progression of aortic stiffness. J Am Coll Cardiol. 2006;47(1):72-75. doi:10.1016/j.jacc.2005.08.052 
24. Chen L, Zhu W, Mai L, Fang L, Ying K. The association of metabolic syndrome and its components with brachial-ankle pulse wave velocity in south China. Atherosclerosis. 2015;240(2):345-350. doi:10.1016/j.atherosclerosis.2015.03.031

25. El Feghali R, Topouchian J, Pannier B, Asmar R. Ageing and blood pressure modulate the relationship between metabolic syndrome and aortic stiffness in never-treated essential hypertensive patients. A comparative study. Diabetes Metab. 2007;33(3):183-188. doi:10.1016/j. diabet.2006.11.011

26. Maloberti A, Bombelli M, Vallerio P, et al. Metabolic syndrome is related to vascular structural alterations but not to functional ones both in hypertensives and healthy subjects. Nutr Metab Cardiovasc Dis. 2021;31(4):1044-1052. doi:10.1016/j.numecd.2020.11.011

27. Maloberti A, Bruno RM, Facchetti R, et al. THE role of metabolic syndrome in blood pressure control and pulse wave velocity progression over a 3.5 years in treated hypertensive PATIENTS. Eur J Intern Med. 2020;76:107-109. doi:10.1016/j.ejim.2020.02.005

28. Kolade OO, O'Moore-Sullivan TM, Stowasser M, et al. Arterial stiffness, central blood pressure and body size in health and disease. Int $J$ Obes. 2012;36(1):93-99. doi:10.1038/ijo.2011.79

29. Mulè G, Cottone S, Mongiovì R, et al. Influence of the metabolic syndrome on aortic stiffness in never treated hypertensive patients. Nutr Metab Cardiovasc Dis. 2006;16(1):54-59. doi:10.1016/j.numecd.2005.03.005

Diabetes, Metabolic Syndrome and Obesity: Targets and Therapy

\section{Dovepress}

\section{Publish your work in this journal}

Diabetes, Metabolic Syndrome and Obesity: Targets and Therapy is an international, peer-reviewed open-access journal committed to the rapid publication of the latest laboratory and clinical findings in the fields of diabetes, metabolic syndrome and obesity research. Original research, review, case reports, hypothesis formation, expert opinion and commentaries are all considered for publication. The manuscript management system is completely online and includes a very quick and fair peer-review system, which is all easy to use. Visit http://www.dovepress. com/testimonials.php to read real quotes from published authors.

Submit your manuscript here: https://www.dovepress.com/diabetes-metabolic-syndrome-and-obesity-targets-and-therapy-journal 